\title{
SPECTROSCOPIC EVIDENCES FOR STAR FORMATION IN COOLING FLOW GALAXIES
}

\author{
N. CARDIEL AND J. GORGAS \\ Dpto. de Astrofísica, Universidad Complutense, Madrid \\ AND \\ A. ARAGON-SALAMANCA \\ Institute of Astronomy, Cambridge, UK
}

$\mathrm{X}$-ray observations have led to the conclusion that many galaxy clusters are hosting cooling flows. The brightest cluster galaxies could have accreted masses of the order of $10^{11}-10^{12} M_{\odot}$, but is still uncertain what the final fate of the accreted gas may be.

Trying to shed some light in the problem, we are carrying out spectroscopic observations of galaxies with and without cooling flows (see details in Cardiel, Gorgas \& Aragón-Salamanca 1995, MNRAS in press). The analysis of $\mathrm{Mg}_{2}$ and the $4000 \AA$ break, good indicators of stellar populations in early-type galaxies, reveals that the nuclear measurements of both indices are well correlated with the mass accretion rate. We conclude that $5-17 \%$ of the mass flow is being converted into new stars with a "normal" IMF. This result is indicative that probably all the gas which is accreted inside the central regions of cooling flow galaxies is being converted into stars.

Our data show that line-strength gradients of galaxies inside large cooling flows flatten in the inner regions $\left(r \leq r_{\mathrm{e}} / 2\right)$, which reveals that the star formation is concentrated toward the central parts. In the mean, $\mathrm{Mg}_{2}$ and the $4000 \AA$ break gradients in cooling flow galaxies are shallower than those found in giant ellipticals. Through the application of stellar population models in the galaxy regions where gradients have presumably been flattened, we derive that the fraction of $V$ light that comes from the accretion population remains roughly constant with radius.

In addition, the indices attained in the outer regions $\left(r>r_{\mathrm{e}}\right)$ by some brightest cluster galaxies, both with and without cooling flows, are significantly lower than those usually found in normal ellipticals. Whether this low indices are indicative of recent star formation processes requires further observational data. 